\title{
Multiple Sign-Changing Solutions for Kirchhoff-Type Equations
}

\author{
Xingping $\mathrm{Li}^{1,2}$ and Xiumei $\mathrm{He}^{3}$ \\ ${ }^{1}$ Department of Mathematics and Statistics, Yunnan University, Kunming, Yunnan 650091, China \\ ${ }^{2}$ Department of Mathematics, Yunnan Normal University, Kunming, Yunnan 650092, China \\ ${ }^{3}$ Department of Mathematics, Kunming University, Kunming, Yunnan 650214, China
}

Correspondence should be addressed to Xiumei He; hexiumei2004@163.com

Received 5 October 2015; Accepted 10 November 2015

Academic Editor: Gabriele Bonanno

Copyright (C) $2015 \mathrm{X}$. Li and X. He. This is an open access article distributed under the Creative Commons Attribution License, which permits unrestricted use, distribution, and reproduction in any medium, provided the original work is properly cited.

We study the following Kirchhoff-type equations $-\left(a+b \int_{\Omega}|\nabla u|^{2} d x\right) \Delta u+V(x) u=f(x, u)$, in $\Omega, u=0$, in $\partial \Omega$, where $\Omega$ is a bounded smooth domain of $\mathbb{R}^{N}(N=1,2,3), a>0, b \geq 0, f \in C(\bar{\Omega} \times \mathbb{R}, \mathbb{R})$, and $V \in C(\bar{\Omega}, \mathbb{R})$. Under some suitable conditions, we prove that the equation has three solutions of mountain pass type: one positive, one negative, and sign-changing. Furthermore, if $f$ is odd with respect to its second variable, this problem has infinitely many sign-changing solutions.

\section{Introduction and Preliminaries}

In this paper, we study the following Kirchhoff-type equations:

$$
\begin{aligned}
-\left(a+b \int_{\Omega}|\nabla u|^{2} d x\right) \Delta u+V(x) u & =f(x, u), \quad \text { in } \Omega, \\
u & =0, \quad \text { on } \partial \Omega,
\end{aligned}
$$

where $\Omega$ is a bounded smooth domain of $\mathbb{R}^{N}(N=1,2,3)$, $a>0, b \geq 0$, and $f \in C(\bar{\Omega} \times \mathbb{R}, \mathbb{R})$ with $F(x, t)=\int_{0}^{t} f(x, s) d s$, $x \in \bar{\Omega}$, and $V \in C(\bar{\Omega}, \mathbb{R})$.

When $V(x)=0$, problem (1) is related to the stationary analogue of the Kirchhoff equation

$$
u_{t t}-\left(a+b \int_{\Omega}|\nabla u|^{2} d x\right) \Delta u=f(x, u),
$$

proposed by Kirchhoff in [1] as an existence of the classical D'Alembert's wave equation for free vibrations of elastic strings. Kirchhoff's model takes into account the changes in length of the string produced by transverse vibrations. Some interesting studies by variational methods can be found in [211]. In these papers, many achievements had been obtained on the existence of ground states, infinitely many radial solutions, soliton solutions, and high energy solution for (1) by using the Fountain Theorem, the mountain pass theorem, using the variational methods and the local minimum methods, and the invariant sets of descent flow. Particularly, in [12], the authors consider the following problem:

$$
\begin{aligned}
-\left(a+b \int_{\Omega}|\nabla u|^{2} d x\right) \Delta u+\phi u & =f(x, u), \quad \text { in } \Omega \\
-\Delta \phi & =u^{2}, \quad \text { in } \Omega \\
u & =0 \quad \text { on } \partial \Omega,
\end{aligned}
$$

where $\Omega$ is a bounded smooth domain of $\mathbb{R}^{N}(N=1,2$ or 3$)$, $a>0, b \geq 0$, and $f: \bar{\Omega} \times \mathbb{R} \rightarrow \mathbb{R}$ is a continuous function which is 3 -superlinear. The unbounded sequence of signchanging solutions of (3) is obtained by using some variants of the mountain pass theorem. In [13], authors considered the following $p$-Laplacian equation coupled with the Dirichlet boundary condition:

$$
\begin{aligned}
-\Delta_{p} u & =\lambda \alpha(x) f(u) \quad \text { in } \Omega, \\
u & =0 \quad \text { on } \partial \Omega,
\end{aligned}
$$

where $p>N$, the parameter $\lambda>0, \alpha \in L^{1}(\Omega)$ is a nonzero potential, and $f \in C([0,+\infty), \mathbb{R})$ with $f(0)=0$. By using variational method, they proved that for every $\lambda>1$ problem (4) has at least two nonzero, nonnegative weak solutions, while there exists $\hat{\lambda}>1$ such that problem (4) has at least 
three nonzero, nonnegative weak solutions. In [14], Ricceri proved that there were at least three distinct weak solutions in $H_{0}^{1}(\Omega)$ for the following equation:

$$
\begin{aligned}
-\Delta u & =\lambda(f(u)+\mu g(u)) \quad \text { in } \Omega \\
u & =0 \quad \text { on } \partial \Omega
\end{aligned}
$$

by using and improving the three critical points' theorem, where $f, g \in C(\mathbb{R}, \mathbb{R})$; let $J_{\mu}$ be an open interval with $J_{\mu} \subset$ $[0,+\infty), \lambda \in J_{\mu}$.

In this paper, we study the sign-changing solutions of problem (1). We need the following assumptions:

$(V) V \in C(\bar{\Omega}, \mathbb{R}), V_{0}:=\inf _{x \in \bar{\Omega}} V(x)>0$.

$\left(f_{1}\right) f \in C(\bar{\Omega} \times \mathbb{R}, \mathbb{R})$, and $|f(x, t)| \leq C\left(1+|t|^{p-1}\right)$ for some $4<p<2^{*}$, where $C$ is a positive constant, $2^{*}=+\infty$ for $N=1,2$, and $2^{*}=6$ for $N=3$.

$\left(f_{2}\right) f(x, t)=o(|t|)$ uniformly in $x \in \bar{\Omega}$, as $|t| \rightarrow 0$.

$\left(f_{3}\right)$ There exist $\mu>4$ and $M>0$ such that

$0<\mu F(x, t) \leq f(x, t) t, \quad \forall(x, t) \in \bar{\Omega} \times \mathbb{R}, \quad|t| \geq M$.

$\left(f_{4}\right) f(x,-t)=-f(x, t)$, for all $x \in \bar{\Omega}$ and $t \in \mathbb{R}$.

We need the following several notations. Let $X:=H_{0}^{1}(\Omega)$ with the inner produce and norm

$$
\begin{aligned}
\langle u, v\rangle & =\int_{\Omega} \nabla u \cdot \nabla v d x \\
\|u\| & =\langle u, u\rangle^{1 / 2}
\end{aligned}
$$

$u, v \in X$

Recall that a function $u \in X$ is called a weak solution of problem (1) if

$$
\begin{aligned}
(a & \left.+b\|u\|^{2}\right) \int_{\Omega} \nabla u \nabla \varphi d x+\int_{\Omega} V(x) u \varphi d x \\
& =\int_{\Omega} f(x, u) \varphi d x, \quad \forall \varphi \in X .
\end{aligned}
$$

Seeking a weak solution of problem (1) is equivalent to finding a critical point of the $C^{1}$-functional

$$
\begin{aligned}
J(u)= & \frac{a}{2} \int_{\Omega}|\nabla u|^{2} d x+\frac{b}{4}\left(\int_{\Omega}|\nabla u|^{2} d x\right)^{2} \\
& +\frac{1}{2} \int_{\Omega} V(x) u^{2} d x-\int_{\Omega} F(x, u) d x, \quad u \in X .
\end{aligned}
$$

Since $\Omega$ is a bounded domain, it is well known that the embedding $X \hookrightarrow L^{s}(\Omega)$ is continuous for all $s \in\left[1,2^{*}\right]$ and the embedding $X \hookrightarrow L^{s}(\Omega)$ is compact for all $s \in\left[1,2^{*}\right)$. Furthermore, there is another norm

$$
\|u\|_{0}=\left(\int_{\Omega}\left(|\nabla u|^{2}+V(x) u^{2}\right) d x\right)^{1 / 2}
$$

and we know that $\|\cdot\|$ and $\|\cdot\|_{0}$ are equivalent on $X$; that is, there exist constants $\underline{C}>0, \bar{C}>0$ such that

$$
\underline{C}\|u\| \leq\|u\|_{0} \leq \bar{C}\|u\|, \quad \forall u \in X .
$$

By Lemma 1 in [9], we know that, under the conditions $(V)$, $\left(f_{1}\right)$, and $\left(f_{2}\right), J \in C^{1}(X, \mathbb{R})$ and for each $u \in X$,

$$
\begin{aligned}
\left\langle J^{\prime}(u), \varphi\right\rangle= & \left(a+b\|u\|^{2}\right) \int_{\Omega} \nabla u \nabla \varphi d x \\
& +\int_{\Omega} V(x) u \varphi d x-\int_{\Omega} f(x, u) \varphi d x,
\end{aligned}
$$

for all $\varphi \in X$.

Our main result of this paper is the following.

Theorem 1. Suppose that $(V)$ and $\left(f_{1}\right)-\left(f_{3}\right)$ are satisfied. Then (1) has three solutions of mountain pass type: one positive, one negative, and one sign-changing. If moreover $f$ is odd with respect to its second variable (i.e., $\left(f_{4}\right)$ holds), then problem (1) has infinitely many sign-changing solutions.

Throughout the paper, $\rightarrow$ and $\rightarrow$ denote the strong and weak convergence, respectively. $C, c, C_{i}$, and $c_{i}$ express distinct constants. For $1 \leq s<\infty$, the usual Lebesgue space is endowed with the norm

$$
\|u\|_{s}:=\left(\int_{\Omega}|u|^{s} d x\right)^{1 / s} .
$$

The paper is organized as follows. In Section 2, we introduce some notions and results of some critical theorem. In Section 3, we complete the proof of Theorem 1.

\section{Some Critical Point Theorems}

Let us begin by recalling some notions and results of some critical point theorems (see [15]).

In the following, $X$ will denote a Hilbert space endowed with the norm $\|\cdot\|_{X}, P \subset X$, which is a closed convex cone.

For $\varepsilon>0$, we denote by $V_{\varepsilon}(S)$ the $\varepsilon$-neighborhood of $S \subset$ $X$; that is,

$$
V_{\varepsilon}(S):=\left\{u \in X: \operatorname{dist}(u, S):=\inf _{v \in S}\|u-v\|_{X}<\varepsilon\right\} .
$$

Define

$$
\begin{aligned}
& +P:=\{u \in X: u \geq 0\}, \\
& -P:=\{u \in X: u \leq 0\}, \\
& P_{\varepsilon}^{ \pm}:=V_{\varepsilon}( \pm P)=\{u \in X: \operatorname{dist}(u, \pm P)<\varepsilon\} .
\end{aligned}
$$

Let $J \in C^{1}(X, \mathbb{R})$. We denote by $K$ the set of critical points of $J$ and $E=X \backslash K$.

For $\varepsilon_{0}>0$, we consider the following situation.

$\left(A_{\varepsilon_{0}}\right)$ : there exists a locally Lipschitz continuous vector field $B: E \rightarrow X$ ( $B$ odd if $J$ is even) such that

(i) $B\left(P_{\varepsilon}^{ \pm} \cap E\right) \subset P_{\varepsilon}^{ \pm}, \forall \varepsilon \in\left(0, \varepsilon_{0}\right)$; 
(ii) there exists a constant $\alpha_{1}>0$ such that

$$
\left\langle J^{\prime}(u), u-B(u)\right\rangle \geq \alpha_{1}\|u-B(u)\|_{X}^{2}, \quad \forall u \in E ;
$$

(iii) for $\rho_{1}<\rho_{2}$ and $\alpha<0$, there exists $\beta>0$ such that $\|u-B(u)\|_{X} \geq \beta$ if $u \in X$ is such that $J(u) \in\left[\rho_{1}, \rho_{2}\right]$ and $\left\|J^{\prime}(u)\right\|_{X^{*}} \geq \alpha$.

Definition 2. Let $J \in C^{1}(X, \mathbb{R}), c \in \mathbb{R}$. One says that $J$ satisfies the $(P S)_{c}$ condition if each sequence $\left\{u_{n}\right\} \subset X$ with $J\left(u_{n}\right) \rightarrow$ $c$ and $J^{\prime}\left(u_{n}\right) \rightarrow 0$ in $X^{*}$ has a convergent subsequence.

Theorem 3 (see $[15])$. Let $J \in C^{1}(X, \mathbb{R})$ with $J(0)=0$. Assume there exists $\varepsilon_{0}>0$ such that $\left(A_{\varepsilon_{0}}\right)$ is satisfied. Assume also that there exist $e_{ \pm} \in \pm P$ and $r>0$ such that

$$
\begin{aligned}
\left(A_{1}\right)\left\|e_{ \pm}\right\|_{X} & >r \\
\rho & :=\inf _{\substack{u \in X \\
\|u\|_{X}=r}} J(u)>\delta:=\max \left\{J(0), J\left(e_{ \pm}\right)\right\} .
\end{aligned}
$$

Then there exist sequences $\left\{u_{ \pm}^{n}\right\} \subset \overline{P_{\varepsilon}^{ \pm}}$such that

$$
\begin{aligned}
& J^{\prime}\left(u_{ \pm}^{n}\right) \longrightarrow 0 \quad \text { in } X^{*}, \\
& J\left(u_{ \pm}^{n}\right) \longrightarrow c_{ \pm}:=\inf _{\gamma \in \Gamma_{ \pm}} \sup _{u \in \gamma([0,1])} J(u) \geq \rho,
\end{aligned}
$$

$$
\forall \varepsilon \in\left(0, \varepsilon_{0}\right)
$$

where

$$
\Gamma_{ \pm}:=\left\{\gamma \in C\left([0,1], \overline{P_{\varepsilon}^{ \pm}}\right): \gamma(0)=0, \gamma(1)=e_{ \pm}\right\} .
$$

If in addition $J$ satisfies the $(P S)_{c}$ condition for any $c>0$, then $J$ has critical point $u_{ \pm} \in \pm P \backslash\{0\}$.

Theorem 4 (see $[15])$. Let $J \in C^{1}(X, \mathbb{R})$. Assume there exists $\varepsilon_{0}>0$ such that $\left(A_{\varepsilon_{0}}\right)$ is satisfied. Assume also that there exists a continuous map $\varphi_{0}: \Delta \rightarrow X$ such that, for any $\varepsilon \in\left(0, \varepsilon_{0}\right)$, the following conditions are satisfied:

(1) $\varphi_{0}\left(\partial_{1} \Delta\right) \subset P_{\varepsilon}^{+}$and $\varphi_{0}\left(\partial_{2} \Delta\right) \subset P_{\varepsilon}^{-}$.

(2) $\varphi_{0}\left(\partial_{0} \Delta\right) \cap P_{\varepsilon}^{+} \cap P_{\varepsilon}^{-}=\varnothing$.

(3) $c_{0}:=\sup _{u \in \varphi_{0}\left(\partial_{0} \Delta\right)} J(u)<c^{*}:=\inf _{u \in \partial P_{\varepsilon}^{+} \bigcap \partial P_{\varepsilon}^{-}} J(u)$,

where

$$
\begin{aligned}
\Delta & =\left\{(s, t) \in \mathbb{R}^{2}: s, t \geq 0, s+t \leq 1\right\}, \\
\partial_{1} \Delta & =\{0\} \times[0,1], \\
\partial_{2} \Delta & =[0,1] \times\{0\}, \\
\partial_{0} \Delta & =\{(s, t) \in \Delta: s+t=1\} .
\end{aligned}
$$

Then there exists a sequence $\left\{u_{n}\right\} \subset \overline{V_{\varepsilon / 2}\left(X \backslash\left(P_{\varepsilon}^{+} \cup P_{\varepsilon}^{-}\right)\right)}$such that

$$
\begin{aligned}
& J^{\prime}\left(u_{n}\right) \longrightarrow 0 \quad \text { in } X^{*}, \\
& J\left(u_{n}\right) \longrightarrow c:=\inf _{\varphi \in \Gamma} \sup _{u \in \varphi(\Delta) \cap\left(X \backslash\left(P_{\varepsilon}^{+} \cup P_{\varepsilon}^{-}\right)\right)} J(u) \geq c_{0},
\end{aligned}
$$

$$
\forall \varepsilon \in\left(0, \varepsilon_{0}\right)
$$

where

$$
\begin{aligned}
\Gamma:= & \left\{\varphi \in C(\Delta, X): \varphi\left(\partial_{1} \Delta\right) \subset P_{\varepsilon}^{+}, \varphi\left(\partial_{2} \Delta\right)\right. \\
& \left.\subset P_{\varepsilon}^{-},\left.\varphi\right|_{\partial_{0} \Delta}=\varphi_{0}\right\} .
\end{aligned}
$$

If in addition $J$ satisfies the $(P S)_{c}$ condition for any $c>0$, then $J$ has a sign-changing critical point.

In the following, we assume that $X$ is of the form

$$
X:=\overline{\bigoplus_{j=1}^{\infty} X_{j}}, \quad \text { with } \operatorname{dim} X_{j}<\infty,
$$

and that there is another norm $\|\cdot\|_{*}$ on $X$ such that $\left(X,\|\cdot\|_{X}\right)$ embeds continuously into $\left(X,\|\cdot\|_{*}\right)$.

We introduce the following notations:

$$
\begin{aligned}
& Y_{k}:=\bigoplus_{j=1}^{k} X_{j}, \\
& Z_{k}:=\overline{\bigoplus_{j=k}^{\infty} X_{j}},
\end{aligned}
$$

for $k \geq 2$,

$$
J^{\alpha}:=\{u \in X: J(u) \leq \alpha\}, \quad \text { for } \alpha \in \mathbb{R} .
$$

Notice that

$$
\begin{aligned}
\left(X,\|\cdot\|_{X}\right) \hookrightarrow\left(X,\|\cdot\|_{*}\right) & \Longrightarrow \exists C_{*}>0, \\
& \text { s.t. }\|u\|_{*} \leq C_{*}\|u\|_{X}, \forall u \in X, \\
\operatorname{dim} Y_{k}<\infty \Longrightarrow \exists \theta_{k} & >0, \\
& \text { s.t. }\|u\|_{X} \leq \theta_{k}\|u\|_{*}, \forall u \in Y_{k} .
\end{aligned}
$$

Assume there exist constants $\rho>0$ and $q>2$ and numbers $\rho_{k}, d_{k}>0$ such that

$$
\frac{\left(\rho_{k} / \theta_{k}\right)^{q}}{\rho_{k}^{2}}+\frac{\rho_{k}\left(\rho_{k} / \theta_{k}\right)}{\rho_{k}+C_{*} d_{k} \rho_{k}}>\rho,
$$

and define

$$
\begin{aligned}
& B_{k}:=\left\{u \in Y_{k}:\|u\| \leq \rho_{k}\right\}, \\
& N_{k}:=\left\{u \in Z_{k}: \frac{\|u\|_{*}^{q}}{\|u\|_{X}^{2}}+\frac{\|u\|_{X} \cdot\|u\|_{*}}{\|u\|_{X}+d_{k} \cdot\|u\|_{*}}=\rho\right\} .
\end{aligned}
$$

In the following, we introduce a sign-changing critical points theorem.

Theorem 5 (see [15]). Let $J \in C^{1}(X, \mathbb{R})$ be an even functional. Assume that there exist $\rho, \rho_{k}, d_{k}>0$ and $q>2$ such that (26) holds. Assume also that there exists $\varepsilon_{0}>0$ such that $\left(A_{\varepsilon_{0}}\right)$ and the following conditions are satisfied:

$\left(B_{1}\right) a_{k}:=\sup _{u \in \partial B_{k}} J(u) \leq 0$ and $b_{k}:=$ $\inf _{u \in N_{k} \cap J^{a_{0}}} J(u) \rightarrow+\infty$, as $k \rightarrow \infty$. 
$\left(B_{2}\right) N_{k} \cap J^{a_{0}} \subset X \backslash\left(P_{\varepsilon}^{+} \cup P_{\varepsilon}^{-}\right), \forall \varepsilon \in\left(0, \varepsilon_{0}\right)$, where $a_{0}:=\max _{u \in B_{k}} J(u)$.

Then, for $k$ large enough there exists a sequence $\left\{u_{k}^{n}\right\}_{n} \subset$ $\overline{V_{\varepsilon / 2}\left(X \backslash\left(P_{\varepsilon}^{+} \cup P_{\varepsilon}^{-}\right)\right)}$such that

$$
\begin{aligned}
& J^{\prime}\left(u_{k}^{n}\right) \longrightarrow 0 \quad \text { in } X^{*} \text { as } n \longrightarrow \infty, \\
& J\left(u_{k}^{n}\right) \longrightarrow c_{k}:=\inf _{\gamma \in \Gamma_{k}} \max _{u \in \gamma\left(B_{k}\right) \cap\left(X \backslash\left(P_{\varepsilon}^{+} \cup P_{\varepsilon}^{-}\right)\right)} J(u) \geq b_{k}, \quad \forall \varepsilon \in\left(0, \varepsilon_{0}\right),
\end{aligned}
$$

where

$$
\begin{aligned}
\Gamma_{k} & :=\left\{\gamma \in C\left(B_{k}, X\right): \gamma \text { is odd, }\left.\gamma\right|_{\partial B_{k}}\right. \\
& \left.=i d \sup _{u \in B_{k}} J(\gamma(u)) \leq a_{0}, \gamma\left(P_{\varepsilon}^{+} \cup P_{\varepsilon}^{-}\right) \subset\left(P_{\varepsilon}^{+} \cup P_{\varepsilon}^{-}\right)\right\} .
\end{aligned}
$$

If in addition $J$ satisfies the $(P S)_{c}$ condition for any $c>0$, then it possesses a sequence $\left\{u_{k}\right\}$ of sign-changing critical points such that $J\left(u_{k}\right) \rightarrow \infty$, as $k \rightarrow \infty$.

\section{Proof of Theorem 1}

We divide the proof of Theorem 1 into the following lemmas.

For $u \in X$ fixed, we consider the functional

$$
\begin{aligned}
\tilde{I}_{u}(v)= & \frac{1}{2}\left(a+b\|u\|^{2}\right) \int_{\Omega}|\nabla v|^{2} d x \\
& +\frac{1}{2} \int_{\Omega} V(x) v^{2} d x-\int_{\Omega} f(x, u) v d x,
\end{aligned}
$$

$v \in X$

It is easy to prove that $\widetilde{I}_{u}$ is of class $C^{1}$, coercive, bounded below, weakly lower semicontinuous, and strictly convex in $X$. Therefore, by Theorem 1.1 in [16], $\widetilde{I}_{u}$ admits a unique global minimizer in $X$ which is the unique solution to the problem

$$
-\left(a+b\|u\|^{2}\right) \Delta v+V(x) v=f(x, u), \quad u \in X .
$$

Here we introduce an auxiliary operator $A$, which will be used to construct the descending flow for the functional $J(\cdot)$. We define an operator $A: X \rightarrow X$ : for $u \in X, A u \in X$ is the unique solution of (31). Then the set of fixed points of $A$ coincide with the set $K$ of critical point of $J$.

Furthermore, the operator $A$ has the following important properties.

Lemma 6. (1) $A$ is continuous and maps bounded sets to bounded sets.

(2) For any $u \in X$, one has

$$
\begin{aligned}
\left\langle J^{\prime}(u), u-A u\right\rangle & \geq a\|u-A u\|^{2}, \\
\left\|J^{\prime}(u)\right\| & \leq\left(a+\bar{C}^{2}+b\|u\|^{2}\right)\|u-A u\|,
\end{aligned}
$$

where $\bar{C}>0$ is defined in (11).

(3) There exists $\varepsilon_{0}>0$ for enough small such that $A\left(P_{\varepsilon}^{ \pm}\right) \subset$ $P_{\varepsilon}^{ \pm}, \forall \varepsilon \in\left(0, \varepsilon_{0}\right)$.
Proof. (1) Let $\left\{u_{n}\right\} \subset X$ such that $u_{n} \rightarrow u$ in $X$. For any $w \in X$, by the definition of $A$, we have

$$
\begin{aligned}
& \left(a+b\left\|u_{n}\right\|^{2}\right) \int_{\Omega} \nabla\left(A u_{n}\right) \nabla w d x \\
& +\int_{\Omega} V(x)\left(A u_{n}\right) w d x=\int_{\Omega} f\left(x, u_{n}\right) w d x \\
& \left(a+b\left\|u_{n}\right\|^{2}\right) \int_{\Omega} \nabla(A u) \nabla w d x \\
& \quad+\int_{\Omega} V(x)(A u) w d x=\int_{\Omega} f(x, u) w d x .
\end{aligned}
$$

Let $v_{n}=A u_{n}$ and $v=A u$. Taking $w=v_{n}-v \in X$ in (33) and (34), we obtain

$$
\begin{aligned}
(a+ & \left.b\left\|u_{n}\right\|^{2}\right)\left\|v_{n}-v\right\|^{2}+\int_{\Omega} V(x)\left(v_{n}-v\right)^{2} d x \\
= & \int_{\Omega}\left[f\left(x, u_{n}\right)-f(x, u)\right]\left(v_{n}-v\right) d x \\
& +b\left(\|u\|^{2}-\left\|u_{n}\right\|^{2}\right) \int_{\Omega} \nabla v \cdot \nabla\left(v_{n}-v\right) d x .
\end{aligned}
$$

Using the Hölder inequality and the Sobolev embedding theorem, we have

$$
\begin{aligned}
\left\|A u_{n}-A u\right\| \leq & C_{1}\left\|f\left(x, u_{n}\right)-f(x, u)\right\|_{p /(p-1)} \\
& +\frac{b}{a}\left|\left\|u_{n}\right\|^{2}-\|u\|^{2}\right| \cdot\|v\|
\end{aligned}
$$

where $C_{1}>0$ is a constant. By $\left(f_{1}\right)$ and Theorem A.1 in [17], one has $f\left(x, u_{n}\right)-f(x, u) \rightarrow 0$ in $L^{p /(p-1)}(\Omega)$. Because $u_{n} \rightarrow$ $u$ in $X$ as $n \rightarrow \infty$, then $\left\|u_{n}\right\| \rightarrow\|u\|$ as $n \rightarrow \infty$. By (36), we obtain $\left\|A u_{n}-A u\right\| \rightarrow 0$ in $X$, which implies that $A$ is continuous on $X$.

On the other hand, for any $u \in X$, taking $w=A u \in X$ in (34), we obtain

$$
\begin{aligned}
(a & \left.+b\|u\|^{2}\right) \int_{\Omega}|\nabla(A u)|^{2} d x+\int_{\Omega} V(x)|A u|^{2} d x \\
& =\int_{\Omega} f(x, u) A u d x .
\end{aligned}
$$

Using the Hölder inequality, the Sobolev embedding theorem, $\left(f_{1}\right)$, and the fact $b \geq 0$, we obtain

$$
\|A u\| \leq C\left(1+\|u\|^{p-1}\right),
$$

where $C>0$ is constant. This shows that $A u$ is bounded in $X$ whenever $u$ is bounded in $X$.

(2) Taking $w=u-A u \in X$ in (34), we have

$$
\begin{gathered}
\left(a+b\|u\|^{2}\right) \int_{\Omega} \nabla(A u) \cdot \nabla(u-A u) d x \\
+\int_{\Omega} V(x) A u \cdot(u-A u) d x \\
=\int_{\Omega} f(x, u)(u-A u) d x ;
\end{gathered}
$$


thus

$$
\begin{aligned}
\left\langle J^{\prime}(u), u-A u\right\rangle= & \left(a+b\|u\|^{2}\right) \int_{\Omega} \nabla u \nabla(u-A u) d x \\
& +\int_{\Omega} V(x) u(u-A u) d x \\
& -\int_{\Omega} f(x, u)(u-A u) d x \\
\geq & a\|u-A u\|^{2}
\end{aligned}
$$

for all $u \in X$. Moreover, using again (34), we have

$$
\begin{aligned}
\left\langle J^{\prime}(u), w\right\rangle= & \left(a+b\|u\|^{2}\right) \int_{\Omega} \nabla u \nabla w d x \\
& +\int_{\Omega} V(x) u w d x-\int_{\Omega} f(x, u) w d x \\
= & \left(a+b\|u\|^{2}\right) \int_{\Omega} \nabla(u-A u) \nabla w d x \\
& +\int_{\Omega} V(x)(u-A u) w d x .
\end{aligned}
$$

By the Hölder inequality, we conclude that

$$
\left\|J^{\prime}(u)\right\| \leq\left(a+\bar{C}^{2}+b\|u\|^{2}\right)\|u-A u\|
$$

where $\bar{C}>0$ is defined in (11).

(3) From $\left(f_{1}\right)$ and $\left(f_{2}\right)$, for any $\varepsilon>0$, there exists $C_{\varepsilon}>0$ such that

$$
\begin{aligned}
& |f(x, t)| \leq \varepsilon|t|+C_{\varepsilon}|t|^{p-1}, \quad \forall t \in \mathbb{R}, \\
& |F(x, t)| \leq \varepsilon|t|^{2}+C_{\varepsilon}|t|^{p}, \quad \forall t \in \mathbb{R} .
\end{aligned}
$$

Set $u \in X$ and $v=A u \in X$. We denote $w^{+}=\max \{0, w\}$ and $w^{-}=\min \{0, w\}$, for any $w \in X$. Taking $w=v^{+}$in (34) and using the Hölder inequality, we obtain

$$
a\left\|v^{+}\right\|^{2} \leq \varepsilon\left\|u^{+}\right\|_{2}\left\|v^{+}\right\|_{2}+C_{\varepsilon}\left\|u^{+}\right\|_{p}^{p-1}\left\|v^{+}\right\|_{p}
$$

which implies

$$
\left\|v^{+}\right\|^{2} \leq \frac{1}{a}\left(\varepsilon\left\|u^{+}\right\|_{2}\left\|v^{+}\right\|_{2}+C_{\varepsilon}\left\|u^{+}\right\|_{p}^{p-1}\left\|v^{+}\right\|_{p}\right) .
$$

Since $\left\|z^{+}\right\|_{s} \leq\|z-w\|_{s}$, for all $z \in X, w \in-P$, and $2 \leq s \leq 2^{*}$, it follows from the Sobolev embedding theorem that there is a constant $C_{1}=C_{1}(s)>0$ such that $\left\|u^{+}\right\|_{s} \leq C_{1} \operatorname{dist}(u,-P)$. Moreover, one can easily verify that $\operatorname{dist}(v,-P) \leq\left\|v^{+}\right\|$. Consequently, by (46) and the Sobolev embedding theorem, we have

$$
\begin{aligned}
& \operatorname{dist}(v,-P)\left\|v^{+}\right\| \leq\left\|v^{+}\right\|^{2} \\
& \quad \leq C_{2}\left[\varepsilon \operatorname{dist}(u,-P)+C_{\varepsilon} \operatorname{dist}(u,-P)^{p-1}\right]\left\|v^{+}\right\|,
\end{aligned}
$$

where $C_{2}>0$. Therefore,

$$
\operatorname{dist}(v,-P) \leq C_{2}\left[\varepsilon \operatorname{dist}(u,-P)+C_{\varepsilon} \operatorname{dist}(u,-P)^{p^{-1}}\right] .
$$

Similarly, we can prove that

$$
\operatorname{dist}(v,+P) \leq C_{3}\left[\varepsilon \operatorname{dist}(u,+P)+C_{\varepsilon} \operatorname{dist}(u,+P)^{p-1}\right] \text {, }
$$

for some constant $C_{3}>0$.

Hence

$$
\operatorname{dist}(v, \pm P) \leq C_{4}\left[\varepsilon \operatorname{dist}(u, \pm P)+C_{\varepsilon} \operatorname{dist}(u, \pm P)^{p-1}\right] \text {, }
$$

where $C_{4}=\max \left\{C_{2}, C_{3}\right\}$. We can choose $\varepsilon_{0}>0$ small enough so that, for all $\varepsilon \in\left(0, \varepsilon_{0}\right)$,

$$
\operatorname{dist}(v, \pm P) \leq \frac{1}{2} \operatorname{dist}(u, \pm P)
$$

$$
\text { whenever } \operatorname{dist}(u, \pm P)<\varepsilon \text {. }
$$

It then follows that $A\left(P_{\varepsilon}^{ \pm}\right) \subset P_{\varepsilon}^{ \pm}, \forall \varepsilon \in\left(0, \varepsilon_{0}\right)$.

Notice that the vector field $A$ is not locally Lipschitz. However, it can be used as [18] to construct a locally Lipschitz vector field which will satisfy condition $\left(A_{\varepsilon_{0}}\right)$. More precisely, we have the following result.

Lemma 7 (see [19, Lemma 3.4]). There exists a locally Lipschitz continuous operator $B: E \triangleq X \backslash K \rightarrow X$ (B odd when $J$ is even) such that

(1) $\left\langle J^{\prime}(u), u-B u\right\rangle \geq(1 / 2)\|u-A u\|^{2}$, for any $u \in E$.

(2) $(1 / 2)\|u-B u\| \leq\|u-A u\| \leq 2\|u-B u\|$, for any $u \in E$.

(3) $B\left(P_{\varepsilon}^{ \pm} \cap E\right) \subset P_{\varepsilon}^{ \pm}$for any $\varepsilon \in\left(0, \varepsilon_{0}\right)$, where $\varepsilon_{0}$ is obtained in Lemma 6(3).

Remark 8. Lemmas 6 and 7 imply that

$$
\begin{aligned}
\left\langle J^{\prime}(u), u-B u\right\rangle & \geq \frac{1}{8}\|u-B u\|^{2}, \\
\left\|J^{\prime}(u)\right\| & \leq 2\left(a+\bar{C}^{2}+b\|u\|^{2}\right)\|u-B u\| .
\end{aligned}
$$

Lemma 9. Let $\rho_{1}<\rho_{2}$ and $\alpha>0$. Then there exists $\beta>0$ such that $\|u-B u\| \geq \beta$ if $u \in X$ is such that $J(u) \in\left[\rho_{1}, \rho_{2}\right]$ and $\left\|J^{\prime}(u)\right\| \geq \alpha$.

Proof. By the definition of the operator A, we have

$$
\begin{aligned}
(a & \left.+b\|u\|^{2}\right) \int_{\Omega} \nabla u \cdot \nabla(A u) d x+\int_{\Omega} V(x) u(A u) d x \\
= & \int_{\Omega} f(x, u) u d x, \quad \forall u \in X .
\end{aligned}
$$


It follows that

$$
\begin{aligned}
J(u) & -\frac{1}{\mu}\left(a+b\|u\|^{2}\right) \int_{\Omega} \nabla u \cdot \nabla(u-A u) d x \\
& -\frac{1}{\mu} \int_{\Omega} V(x) u(u-A u) d x \\
= & {\left[a\left(\frac{1}{2}-\frac{1}{\mu}\right)+b\left(\frac{1}{4}-\frac{1}{\mu}\right)\|u\|^{2}\right] \cdot\|u\|^{2} } \\
+ & \left(\frac{1}{2}-\frac{1}{\mu}\right) \int_{\Omega} V(x) u^{2} d x \\
+ & \int_{\Omega}\left[\frac{1}{\mu} f(x, u) u-F(x, u)\right] d x \\
\geq & a\left(\frac{1}{2}-\frac{1}{\mu}\right)\|u\|^{2}+b\left(\frac{1}{4}-\frac{1}{\mu}\right)\|u\|^{4} .
\end{aligned}
$$
has

If $b>0$, using Lemma 7(2) and the Hölder inequality, one

$$
\begin{aligned}
& a\left(\frac{1}{2}-\frac{1}{\mu}\right)\|u\|^{2}+b\left(\frac{1}{4}-\frac{1}{\mu}\right)\|u\|^{4} \\
& \quad \leq|J(u)|+\frac{1}{\mu}\left(a+\bar{C}^{2}+b\|u\|^{2}\right)\|u\| \cdot\|u-A u\| \\
& \quad \leq|J(u)|+\frac{2}{\mu}\left(a+\bar{C}^{2}+b\|u\|^{2}\right)\|u\| \cdot\|u-B u\|,
\end{aligned}
$$

for $\bar{C}>0$ (see (11)). Suppose that there exists a sequence $\left\{u_{n}\right\} \subset X$ such that $J\left(u_{n}\right) \in\left[\rho_{1}, \rho_{2}\right],\left\|J^{\prime}\left(u_{n}\right)\right\| \geq \alpha$ and $\left\|u_{n}-B u_{n}\right\| \rightarrow 0$. By (55), we see that $\left\{\left\|u_{n}\right\|\right\}$ is bounded. It follows from Remark 8 above that $\left\|J^{\prime}\left(u_{n}\right)\right\| \rightarrow 0$, which is a contradiction.

If $b=0$, the conclusion is concluded by Remark 8 .

Lemma 10. The functional J satisfies the (PS) condition at any level $c \in \mathbb{R}$.

Proof. In view of (9) and (12), $\left(f_{3}\right)$, for any $u \in X$, one has

$$
\begin{aligned}
J(u) & -\frac{1}{\mu}\left\langle J^{\prime}(u), u\right\rangle \\
= & a\left(\frac{1}{2}-\frac{1}{\mu}\right)\|u\|^{2}+b\left(\frac{1}{4}-\frac{1}{\mu}\right)\|u\|^{4} \\
& +\left(\frac{1}{2}-\frac{1}{\mu}\right) \int_{\Omega} V(x) u^{2} d x \\
& +\int_{\Omega}\left[\frac{1}{\mu} f(x, u) u-F(x, u)\right] d x \\
\geq & a\left(\frac{1}{2}-\frac{1}{\mu}\right)\|u\|^{2} .
\end{aligned}
$$

Let $\left\{u_{n}\right\} \subset X$ be a sequence such that $\sup _{n}\left|J\left(u_{n}\right)\right|<\infty$ and $J^{\prime}\left(u_{n}\right) \rightarrow 0$ in $X^{*}$ as $n \rightarrow \infty$. Inequality (56) implies that $\left\{u_{n}\right\}$ is bounded in $X$. Then, up to a subsequence, we have $u_{n} \rightarrow u$ in $X$ and $u_{n} \rightarrow u L^{s}(\Omega)$ for $2 \leq s<2^{*}$. Using a standard argument, one has $J^{\prime}(u)=0$. Notice that

$$
\begin{aligned}
o_{n}(1)= & \left\langle J^{\prime}\left(u_{n}\right)-J^{\prime}(u), u_{n}-u\right\rangle \\
= & \left(a+b\|u\|^{2}\right) \int_{\Omega}\left|\nabla\left(u_{n}-u\right)\right|^{2} d x \\
& +b\left(\left\|u_{n}\right\|^{2}-\|u\|^{2}\right) \int_{\Omega} \nabla u \cdot \nabla\left(u_{n}-u\right) d x \\
& +\int_{\Omega} V(x)\left(u_{n}-u\right)^{2} d x \\
& -\int_{\Omega}\left[f\left(x, u_{n}\right)-f(x, u)\right]\left(u_{n}-u\right) d x \\
\geq & a\left\|u_{n}-u\right\|^{2}+b\left(\left\|u_{n}\right\|^{2}-\|u\|^{2}\right) \int_{\Omega} \nabla u \\
& \cdot \nabla\left(u_{n}-u\right) d x \\
& -\int_{\Omega}\left[f\left(x, u_{n}\right)-f(x, u)\right]\left(u_{n}-u\right) d x .
\end{aligned}
$$

Consequently, by (43), the Hölder inequality, and the boundness of $\left\{u_{n}\right\}$ in $X$, we know that, for any $\varepsilon>0$, there is a constant $C_{\varepsilon}>0$ such that

$$
\begin{aligned}
a\left\|u_{n}-u\right\|^{2} \leq & \varepsilon\left\|u_{n}\right\|_{2} \cdot\left\|u_{n}-u\right\|_{2}+C_{\varepsilon}\left\|u_{n}\right\|_{p}^{p-1} \\
& \cdot\left\|u_{n}-u\right\|_{p}+\varepsilon\|u\|_{2} \cdot\left\|u_{n}-u\right\|_{2} \\
& +C_{\varepsilon}\|u\|_{p}^{p-1} \cdot\left\|u_{n}-u\right\|_{p}+o_{n}(1) .
\end{aligned}
$$

By the arbitrariness of $\varepsilon$ and $X \hookrightarrow L^{s}(\Omega)$ which is compact for $s \in\left[2,2^{*}\right)$, we have $u_{n} \rightarrow u$ in $X$.

Lemma 11. For $s \in\left[1,2^{*}\right]$, there exists $k>0$ such that for any $\varepsilon>0$

$$
\|u\|_{s} \leq k \varepsilon, \quad \forall u \in P_{\varepsilon}^{+} \cap P_{\varepsilon}^{-} .
$$

Proof. For any $u \in X$, this follows from the fact that

$$
\begin{aligned}
\left\|u^{ \pm}\right\|_{s} & =\inf _{w \in \mp P}\|u-w\|_{s} \leq C_{s} \inf _{w \in \mp P}\|u-w\| \\
& =C_{s} \operatorname{dist}(u, \mp P),
\end{aligned}
$$

where $C_{s}>0$ is the Sobolev constant in the continuous embedding $X \hookrightarrow L^{s}(\Omega)$ for all $s \in\left[1,2^{*}\right]$.

Lemma 12. For $\varepsilon>0$ small enough, one has

$$
J(u) \geq \frac{a}{8} \varepsilon^{2}, \quad \forall u \in \partial P_{\varepsilon}^{+} \cap \partial P_{\varepsilon}^{-} .
$$

Proof. Let $u \in \partial P_{\varepsilon}^{+} \cap \partial P_{\varepsilon}^{-}$. It is clear that

$$
\left\|u^{ \pm}\right\| \geq \operatorname{dist}(u, \mp P)=\varepsilon, \quad \forall \varepsilon>0 .
$$

Using Lemma 11, we obtain

$$
J(u) \geq \frac{a}{4} \varepsilon^{2}-C \varepsilon^{p},
$$

where $C>0$. 
Let us denote by $0<\lambda_{1}<\lambda_{2}<\lambda_{3} \leq \cdots$ the distinct eigenvalues of the problem

$$
\begin{aligned}
-\Delta u & =\lambda u & & \text { in } \Omega, \\
u & =0, & & \text { on } \partial \Omega .
\end{aligned}
$$

It is well known that each $\lambda_{j}(j \geq 2)$ has finite multiplicity, the principle eigenvalue $\lambda_{1}$ is simple with positive eigenfunction $e_{1}$, and the eigenfunctions $e_{j}$ corresponding to $\lambda_{j}(j \geq 2)$ are sign-changing. Let $X_{j}$ be the eigenspace associated with $\lambda_{j}$. We set $k \geq 2$

$$
\begin{aligned}
Y_{k}: & =\bigoplus_{j=1}^{k} X_{j}, \\
Z_{k}: & =\overline{\bigoplus_{j=k}^{\infty} X_{j} .}
\end{aligned}
$$

Note that any element of $Z_{k} \backslash\{0\}$ is sign-changing.

We define

$$
N_{k}:=\left\{u \in Z_{k}: \frac{\|u\|_{p}^{p}}{\|u\|^{2}}+\frac{\|u\| \cdot\|u\|_{p}}{\|u\|+\beta_{k}^{-\sigma} \cdot\|u\|_{p}}=\rho\right\},
$$

where

$$
\begin{aligned}
\rho & :=\frac{a}{8 C_{\varepsilon}}, \quad C_{\varepsilon} \text { is obtained in (43), } \\
\beta_{k} & :=\sup _{\substack{u \in Z_{k} \\
\|u\|=1}}\|u\|_{p}, \\
\sigma & :=\frac{2}{p(p-2)} .
\end{aligned}
$$

Lemma 13. One has for any fixed $\alpha>0$

$$
\inf _{u \in N_{k} \cap J^{\alpha}} J(u) \longrightarrow+\infty, \quad k \longrightarrow \infty .
$$

Proof. By the definition $N_{k}$, we have

$$
\frac{\|u\|_{p}^{p}}{\|u\|^{2}} \leq \rho, \quad \forall u \in N_{k} .
$$

For each fixed $u \in N_{k}$, for $\varepsilon>0$ small enough, by (44) and (67), we have

$$
\begin{aligned}
J(u) & \geq \frac{a}{4}\|u\|^{2}-C_{\varepsilon}\|u\|_{p}^{p}=\|u\|^{2}\left(\frac{a}{4}-C_{\varepsilon} \frac{\|u\|_{p}^{p}}{\|u\|^{2}}\right) \\
& \geq \frac{a}{8}\|u\|^{2} .
\end{aligned}
$$

Notice that

$$
\|u\|_{p} \leq \rho\left(1+C_{p} \beta_{k}^{-\sigma}\right), \quad \forall u \in N_{k},
$$

where $C_{p}>0$ is the Sobolev constant in the embedding $X \hookrightarrow$ $L^{p}(\Omega)$.
For any $u \in N_{k} \cap J^{\alpha}$, one has

$$
\frac{a}{4}\|u\|^{2} \leq \alpha+C\|u\|_{p}^{p} .
$$

This implies that

$$
\begin{aligned}
&\|u\| \leq C_{*}:=C(\alpha, p, \rho)\left(1+\beta_{k}^{-(\sigma / 2) p}\right), \\
& \forall u \in N_{k} \cap J^{\alpha},
\end{aligned}
$$

where $C(\alpha, p, \rho)>0$.

Furthermore, $\forall u \in N_{k} \cap J^{\alpha}$, one has

$$
\begin{aligned}
\rho & =\frac{\|u\|_{p}^{p}}{\|u\|^{2}}+\frac{\|u\| \cdot\|u\|_{p}}{\|u\|+\beta_{k}^{-\sigma}\|u\|_{p}} \leq \frac{\|u\|_{p}^{p}}{\|u\|^{2}} \\
& +\frac{\|u\| \cdot\|u\|_{p}}{2\left(\|u\| \cdot \beta_{k}^{-\sigma}\|u\|_{p}\right)^{1 / 2}}=\left(\frac{\|u\|_{p}}{\|u\|}\right)^{2}\|u\|_{p}^{p-2} \\
& +\frac{1}{2}\left(\|u\| \cdot \beta_{k}^{\sigma} \cdot\|u\|_{p}\right)^{1 / 2} \leq \beta_{k}^{2} \cdot C_{p}^{p-2} \cdot\|u\|^{p-2} \\
& +\frac{\sqrt{C_{p}}}{2} \cdot \beta_{k}^{\sigma / 2} \cdot\|u\|=\beta_{k}^{2} \cdot C_{p}^{p-2} \cdot C_{*}^{p-2} \cdot\left\|\frac{1}{C_{*}} u\right\|^{p-2} \\
& +\frac{\sqrt{C_{p}}}{2} \cdot \beta_{k}^{\sigma / 2} \cdot C_{*} \cdot\left\|\frac{1}{C_{*}} u\right\| \\
& \leq\left(\beta_{k}^{2} C_{p}^{p-2} C_{*}^{p-2}+\frac{\sqrt{C_{p}}}{2} \beta_{k}^{\sigma / 2} C_{*}\right) \\
& \cdot \max \left\{\left\|\frac{1}{C_{*}} u\right\|,\left\|\frac{1}{C_{*}} u\right\|^{p-2}\right\},
\end{aligned}
$$

where $C_{p}>0$ is the Sobolev constant in the embedding $X \hookrightarrow$ $L^{p}(\Omega)$. By $(74)$, one has $\left\|\left(1 / C_{*}\right) u\right\| \leq 1$, for $u \in N_{k} \cap J^{\alpha}$. Hence,

$$
\begin{aligned}
& \max \left\{\left\|\frac{1}{C_{*}} u\right\|,\left\|\frac{1}{C_{*}} u\right\|^{p-2}\right\}=\left\|\frac{1}{C_{*}} u\right\|, \\
& \rho \leq\left(\beta_{k}^{2} C_{p}^{p-2} C_{*}^{p-3}+\frac{\sqrt{C_{p}}}{2} \beta_{k}^{\sigma / 2}\right)\|u\|,
\end{aligned}
$$

$\forall u \in N_{k} \cap J^{\alpha}$.

For any $u \in N_{k} \cap J^{\alpha}$, we deduce

$$
\rho \leq \widetilde{C}(\alpha, p, \rho)\left(\beta_{k}^{2}+\beta_{k}^{\sigma / 2}+\beta_{k}^{2-(\sigma / 2) p(p-2)}\right)\|u\|,
$$

where $\widetilde{C}(\alpha, p, \rho)>0$ is a constant. From (71), one has

$$
\begin{aligned}
J(u) \geq & \frac{a}{8} \rho^{2} \cdot \widetilde{C}(\alpha, p, \rho)^{-2} \\
& \cdot\left(\beta_{k}^{2}+\beta_{k}^{\sigma / 2}+\beta_{k}^{2-(\sigma / 2) p(p-2)}\right)^{-2},
\end{aligned}
$$


By (68), one has $2-(\sigma / 2) p(p-2)=1>0$. From Lemma 3.8 in [17], we know that $\beta_{k} \rightarrow 0$, as $k \rightarrow \infty$. Set $k \rightarrow \infty$ in (78); we have

$$
\inf _{u \in N_{k} \cap J^{\alpha}} J(u) \longrightarrow+\infty, \quad \text { as } k \longrightarrow \infty .
$$

Lemma 14. For any $\alpha>0$, one has

$$
\delta_{0}(\alpha):=\operatorname{dist}\left(N_{k} \cap J^{\alpha}, P\right)>0 .
$$

Proof. The proof is similar to the proof of Lemma 5.4 in [20].

\section{Proof of Theorem 1 .}

Step 1 (the existence of a positive and a negative solution). By (44) and the Sobolev embedding theorem, $\forall 0<\varepsilon<(1 / 4) a$, there exists constant $C>0$ such that

$$
J(u) \geq \frac{a}{4}\|u\|^{2}-C\|u\|^{p} .
$$

Consequently, there exists $r>0$ (small enough) such that

$$
\inf _{\|u\|=r} J(u) \geq \frac{1}{8} r^{2}>0 .
$$

From $\left(f_{3}\right)$ and (44), we have

$$
F(x, t) \geq C_{1}|t|^{\mu}-C_{2} t^{2}, \quad \forall(x, t) \in \bar{\Omega} \times \mathbb{R},
$$

for some positive constants $C_{1}$ and $C_{2}$. Thus, by (83) and the Sobolev embedding theorem, one has

$$
J(u) \leq C_{3}\|u\|^{2}+\frac{b}{4}\|u\|^{4}-C_{4}\|u\|_{\mu}^{\mu},
$$

where $C_{3}>0, C_{4}>0$. Fixing $e \in X \backslash\{0\}$, it is easy to prove that

$$
J(t e) \longrightarrow-\infty \quad \text { as } t \longrightarrow+\infty \text {. }
$$

Therefore, we can find $e_{ \pm} \in \pm P$ such that

$$
\begin{gathered}
\left\|e_{ \pm}\right\|>r \\
J\left(e_{ \pm}\right)<0 .
\end{gathered}
$$

This shows that condition $\left(A_{1}\right)$ of Theorem 3 is satisfied. By Lemmas 6, 7, and 9, condition $\left(A_{\varepsilon_{0}}\right)$ is satisfied for $\varepsilon_{0}>0$ small enough. By Lemma 10, $J$ satisfies the $(P S)$ condition at any positive level $c$. Hence, the existence of a positive and a negative solution follows from Theorem 3 .

In the following proof, we adopt the notations of Theorem 4.

Step 2 (the existence of a sign-changing solution). Using the the main idea of [21], we will verify the assumptions of Theorem 4.

Let $v_{1}, v_{2} \in C_{0}^{\infty}(\Omega) \backslash\{0\}$ be such that $\operatorname{supp}\left(v_{1}\right) \cap \operatorname{supp}\left(v_{2}\right)=$ $\varnothing, v_{1} \leq 0$ and $v_{2} \geq 0$. We define the continuous map $\varphi_{0}: \Delta \rightarrow$ $X$ by $\varphi_{0}(s, t)=R\left(s v_{1}+t v_{2}\right)$ for all $(s, t) \in \Delta$, where $R>0$ is a constant to be determined later. Obviously, $\varphi_{0}(0, t) \in P_{\varepsilon}^{+}$ and $\varphi_{0}(s, 0) \in P_{\varepsilon}^{-}$for all $\varepsilon>0$. This implies that $\varphi_{0}\left(\partial_{1} \Delta\right) \subset P_{\varepsilon}^{+}$ and $\varphi_{0}\left(\partial_{2} \Delta\right) \subset P_{\varepsilon}^{-}$; that is, Theorem $4(1)$ holds. Now a simple computation is as follows:

$$
\delta:=\min \left\{\left\|(1-t) v_{1}+t v_{2}\right\|_{2}: t \in[0,1]\right\}>0 .
$$

Then $\|u\|_{2} \geq \delta R$ for $u \in \varphi_{0}\left(\partial_{0} \Delta\right)$ and it follows from Lemma 11 that $\varphi_{0}\left(\partial_{0} \Delta\right) \cap P_{\varepsilon}^{+} \cap P_{\varepsilon}^{-}=\varnothing$, for $R$ large enough and for any $\varepsilon>0$.

By (84), there exist constants $C_{3}>0$ and $C_{4}>0$ such that

$$
J(u) \leq C_{3}\|u\|^{2}+\frac{b}{4}\|u\|^{4}-C_{4}\|u\|_{\mu}^{\mu} .
$$

Combining with Lemma 12, for $R$ large enough and $\varepsilon>0$ small enough, we obtain

$$
c_{0}=\sup _{u \in \varphi_{0}\left(\partial_{0} \Delta\right)} J(u)<0<c^{*}:=\inf _{u \in \partial P_{\varepsilon}^{+} \cap \partial P_{\varepsilon}^{-}} J(u) .
$$

By Theorem $4, J$ has a sign-changing critical point.

Step 3 (the existence of infinitely many high energy solutions). Because $\operatorname{dim} Y_{k}<+\infty$, there exists $\theta_{k}>0$ such that $\|u\| \leq$ $\theta_{k}\|u\|_{\mu}$ for any $u \in Y_{k}$. Combining with (83), one has

$$
J(u) \leq C_{1}\|u\|^{2}+\frac{b}{4}\|u\|^{4}-C_{2}\|u\|^{\mu}, \quad \forall u \in Y_{k},
$$

where $C_{1}>0, C_{2}>0$. Hence, we have $J(u) \rightarrow-\infty$ on $Y_{k}$ as $\|u\| \rightarrow \infty$.

We can then choose $\rho_{k}>0$ large enough so that

$$
\begin{gathered}
\frac{\left(\rho_{k} / \theta_{k}\right)^{p}}{\rho_{k}^{2}}+\frac{\rho_{k}\left(\rho_{k} / \theta_{k}\right)}{\rho_{k}+C_{p} \beta_{k}^{-\sigma} \rho_{k}}>\rho, \\
a_{k}:=\max _{\substack{u \in Y_{k} \\
\|u\|=\rho_{k}}} J(u)<0,
\end{gathered}
$$

where $\sigma$ is given by (67) and $C_{p}$ is the Sobolev constant. Combining with Lemma 13 , condition $\left(B_{1}\right)$ of Theorem 5 is satisfied. By Lemma 14,

$$
\delta_{0}\left(a_{0}\right):=\operatorname{dist}\left(N_{k} \cap J^{a_{0}}, P\right)>0,
$$

where $a_{0}:=\max _{u \in B_{k}} J(u)>0$.

For any $u \in N_{k} \cap J^{a_{0}}, v \in P$, and $w \in P_{\varepsilon}^{+} \cap P_{\varepsilon}^{-}$, one has

$$
\begin{aligned}
0 & <\delta_{0}\left(a_{0}\right)=\inf _{\substack{u \in N_{k} \cap J^{a_{0}} \\
v \in P}}\|u-v\| \\
& \leq\|u-w\|+\left\|w^{+}-v^{+}\right\|+\left\|w^{-}-v^{-}\right\|,
\end{aligned}
$$

where $v^{+}=\max \{v, 0\}, v^{-}=\min \{v, 0\}, w^{+} \in P_{\varepsilon}^{+}$, and $w^{-} \in P_{\varepsilon}^{-}$. Hence

$$
0<\delta_{0}\left(a_{0}\right)=\operatorname{dist}\left(u, P_{\varepsilon}^{+} \cup P_{\varepsilon}^{-}\right)+2 \varepsilon .
$$

Set $\varepsilon_{0} \in\left(0,(1 / 2) \delta_{0}\left(a_{0}\right)\right)$; one has

$$
\operatorname{dist}\left(u, P_{\varepsilon}^{+} \cup P_{\varepsilon}^{-}\right) \geq \delta_{0}\left(a_{0}\right)-2 \varepsilon>0, \quad \forall \varepsilon \in\left(0, \varepsilon_{0}\right) .
$$

This implies that condition $\left(B_{2}\right)$ of Theorem 5 holds. Thus, by Theorem 5 , we obtain that $J$ possess a sequence $\left\{u_{k}\right\}$ of signchanging critical point such that $J\left(u_{k}\right) \rightarrow \infty$ as $k \rightarrow \infty$. 


\section{Conflict of Interests}

The authors declare that there is no conflict of interests regarding the publication of this paper.

\section{Acknowledgment}

This work is supported by Scientific Research Fund of Yunnan Province Education Department (2015C075Y).

\section{References}

[1] G. Kirchhoff, Mechanik, Teubner, Leipzig, Germany, 1883.

[2] C. O. Alves, F. J. S. A. Corrêa, and T. F. Ma, "Positive solutions for a quasilinear elliptic equation of Kirchhoff type," Computers \& Mathematics with Applications, vol. 49, no. 1, pp. 85-93, 2005.

[3] X.-M. He and W.-M. Zou, "Multiplicity of solutions for a class of Kirchhoff type problems," Acta Mathematicae Applicatae Sinica, English Series, vol. 26, no. 3, pp. 387-394, 2010.

[4] J. H. Jin and X. Wu, "Infinitely many radial solutions for Kirchhoff-type problems in RN," Journal of Mathematical Analysis and Applications, vol. 369, no. 2, pp. 564-574, 2010.

[5] W. Liu and X. M. He, "Multiplicity of high energy solutions for superlinear Kirchhoff equations," Journal of Applied Mathematics and Computing, vol. 39, no. 1-2, pp. 473-487, 2012.

[6] Q. Q. Li and X. Wu, "A new result on high energy solutions for Schrödinger-Kirchhoff type equations in $\mathbb{R}^{N}$," Applied Mathematics Letters, vol. 30, pp. 24-27, 2014.

[7] A. Mao and Z. Zhang, "Sign-changing and multiple solutions of Kirchhoff type problems without the P.S. condition," Nonlinear Analysis: Theory, Methods \& Applications, vol. 70, no. 3, pp. 1275-1287, 2009.

[8] J. J. Nie, "Existence and multiplicity of nontrivial solutions for a class of Schrödinger-Kirchhoff-type equations," Journal of Mathematical Analysis and Applications, vol. 417, no. 1, pp. 6579, 2014.

[9] X. Wu, "Existence of nontrivial solutions and high energy solutions for Schrödinger-Kirchhoff-type equations in $\mathbb{R}^{N}$," Nonlinear Analysis: Real World Applications, vol. 12, no. 2, pp. 1278-1287, 2011.

[10] X. Wu, "High energy solutions of system of Kirchhoff-type equations in $\mathbb{R}^{N}$," Journal of Mathematical Physics, vol. 53, Article ID 063508, 2012.

[11] Z. Zhang and K. Perera, "Sign changing solutions of Kirchhoff type problems via invariant sets of descent flow," Journal of Mathematical Analysis and Applications, vol. 317, no. 2, pp. 456463, 2006.

[12] C. J. Batkam and J. Júnior, "Schrödinger-Kirchhoff-Poisson type systems," http://arxiv.org/abs/1503.07280.

[13] F. Faraci and A. Kristály, "Three non-zero solutions for a nonlinear eigenvalue problem," Journal of Mathematical Analysis and Applications, vol. 394, no. 1, pp. 225-230, 2012.

[14] B. Ricceri, "On a three critical points theorem," Archiv der Mathematik, vol. 75, no. 3, pp. 220-226, 2000.

[15] C. J. Batkam, "An elliptic equation under the effect of two nonlocal terms," Mathematical Methods in the Applied Sciences, 2015.

[16] J. Mawhin and M. Willem, Critical Point Theory and Hamiltonian System, vol. 74 of Applied Mathematical Sciences, Springer, New York, NY, USA, 1989.
[17] M. Willem, Minimax Theorems, Birkhäuser, Boston, Mass, USA, 1996.

[18] T. Bartsch and Z. Liu, "Multiple sign changing solutions of a quasilinear elliptic eigenvalue problem involving the p-Laplacian," Communications in Contemporary Mathematics, vol. 6, no. 2, pp. 245-258, 2004.

[19] C. J. Batkam, "Multiple sign-changing solutions to a class of Kirchhoff type problems," http://arxiv.org/abs/1501.05733.

[20] W. M. Zou, Sign-Changing Critical Point Theory, Springer, New York, NY, USA, 2008.

[21] Z. Liu, Z.-Q. Wang, and J. Zhang, "Infinitely many sign-changing solutions for the nonlinear Schrödinger-Poisson system," Annali di Matematica Pura ed Applicata, 2015. 


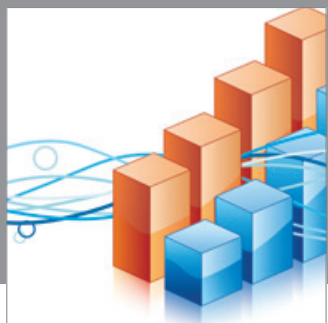

Advances in

Operations Research

mansans

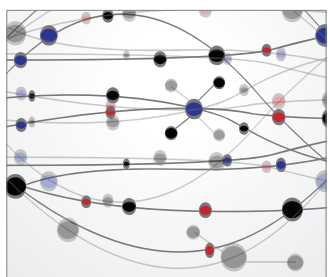

The Scientific World Journal
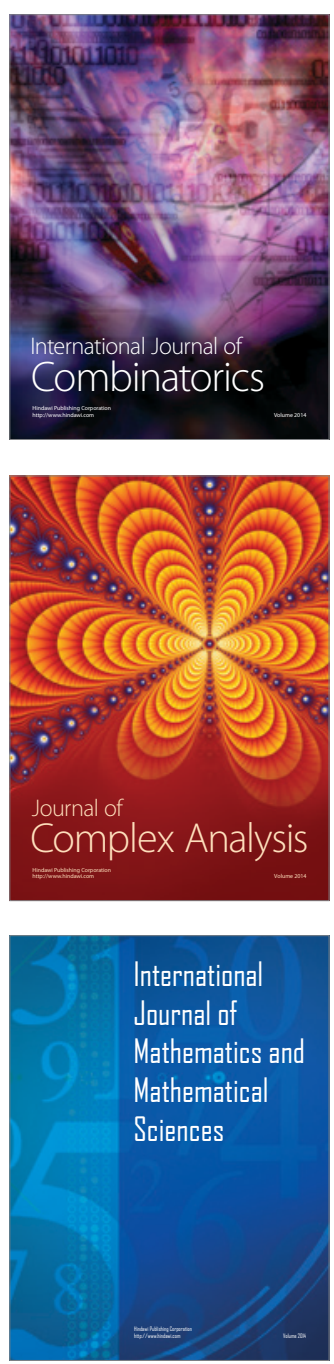
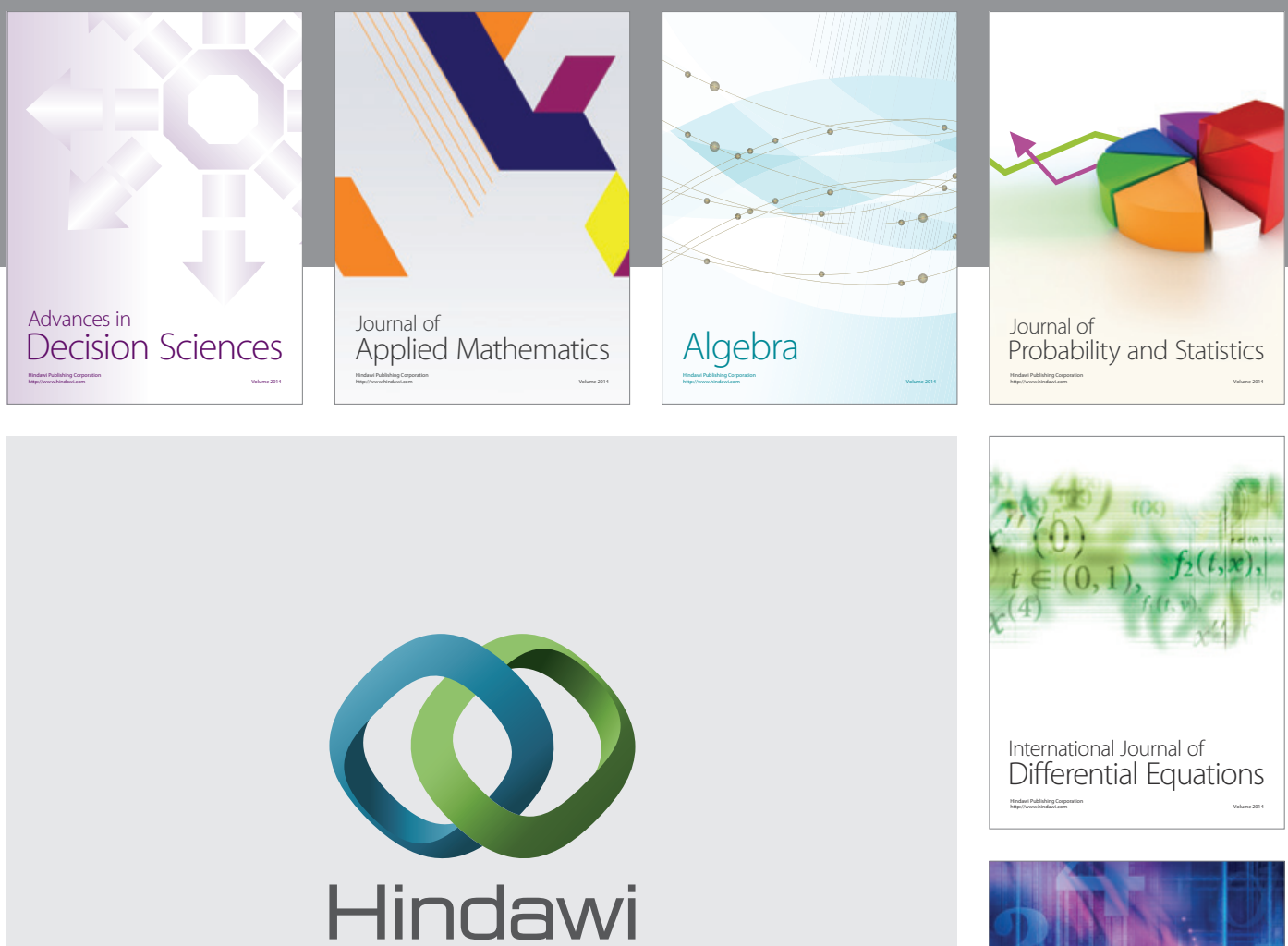

Submit your manuscripts at http://www.hindawi.com
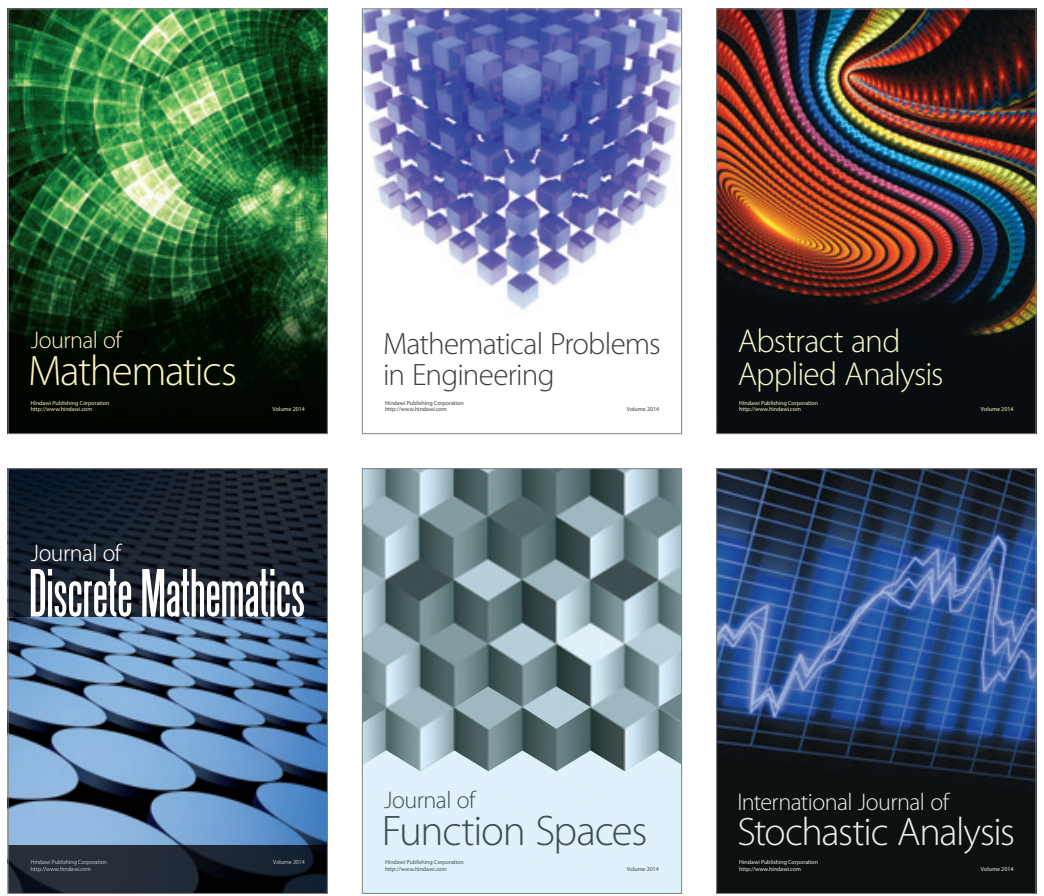

Journal of

Function Spaces

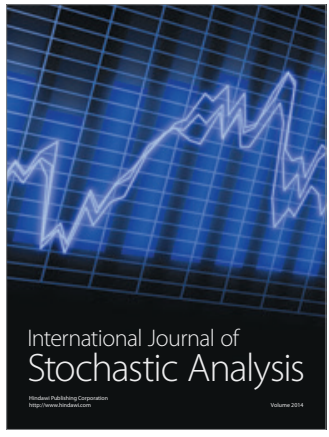

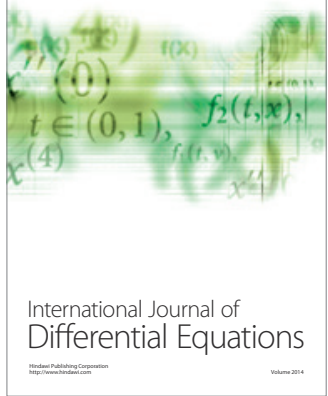
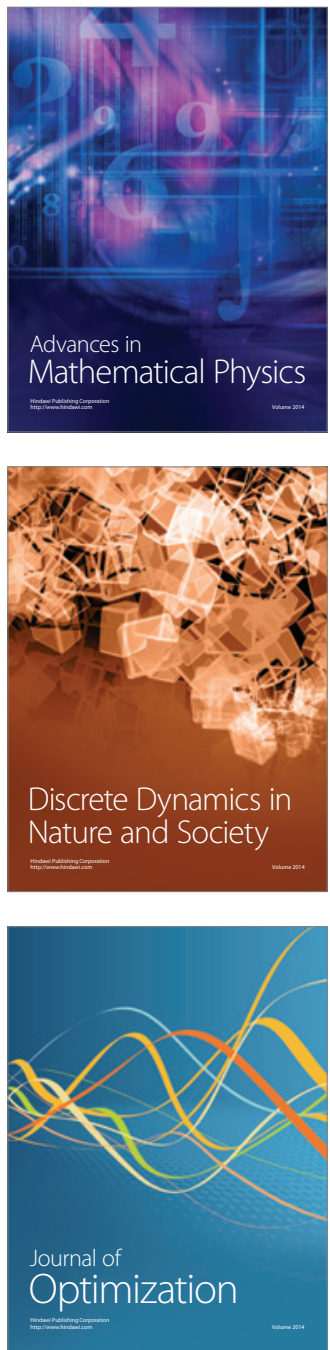